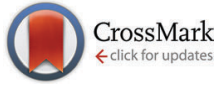

Cite this: Phys. Chem. Chem. Phys., $2016,18,7148$

Received 13th November 2015, Accepted 21st January 2016

DOI: $10.1039 / \mathrm{c} 5 \mathrm{cp} 06971 \mathrm{~h}$

www.rsc.org/pccp

\title{
Ultrafast deactivation of bilirubin: dark intermediates and two-photon isomerization $\dagger$
}

\author{
Carlos Carreira-Blanco, ${ }^{a}$ Patrick Singer, ${ }^{b}$ Rolf Diller ${ }^{\star b}$ and J. Luis Pérez Lustres ${ }^{{ }^{a}}$
}

Bilirubin is a neurotoxic product responsible for neonatal jaundice, which is generally treated by phototherapy. The photoreaction involves ultrafast internal conversion via an elusive intermediate and $Z-E$ isomerization with minor yield (less than $3 \%$ in solution). The structure of the intermediate remains unclear. Here, the combination of UV-vis and mid-IR ultrafast transient absorption spectroscopy reports a comprehensive picture of the mechanism and provides essential structural information about the intermediate species. Thus, spectral dynamics during the earliest ps unveils a wavepacket travelling from the Franck-Condon region to the crossing point with a dark state. The latter shows a tighter molecular skeleton than the ground state and decays with 15 ps time constant. Remarkably, the relative contribution of a non-decaying component increases linearly with pump energy, suggesting that $Z-E$ isomerization could also be triggered by two-photon excitation. Implications for the photochemistry of protein-bound open tetrapyrroles are discussed.

\section{Introduction}

Tetrapyrroles constitute arguably one of the most relevant classes of molecules. Porphyrins, closed chain tetrapyrroles, are essential for oxygen transport and photosynthesis, whereas open chain bilins sense and trigger the biological response to light in phytochromes. $^{1,2}$ It is fascinating that this relatively simple structure fulfils effectively various essential functions in a vast variety of living organisms. This has inspired applications from solar energy conversion ${ }^{3-5}$ to cancer treatment. ${ }^{6}$ We focus on bilirubin, a linear chain tetrapyrrole resulting from the degradation of the heme group.'

Bilirubin IX $\alpha$ (BR) consists of two dipyrrinone units connected by a methylene group, Scheme 1 . The substitution pattern of the pyrroles is crucial for the $3 \mathrm{D}$ structure. ${ }^{8,9}$ Thus, intramolecular hydrogen bonding between the carboxylic groups and the terminal

\footnotetext{
${ }^{a}$ Centro Singular de Investigación en Química Biolóxica e Materiais Moleculares (CIQUS) and Department of Physical Chemistry, Universidade de Santiago de Compostela, C/Jenaro de la Fuente s/n, E-15782 Santiago de Compostela, Spain. E-mail: luis.lustres@usc.es; Fax: +348818 15704; Tel: +34 881815722

${ }^{b}$ Fachbereich Physik, Technische Universität Kaiserslautern, Erwin Schrödinger Straße, Building 46, D-67663 Kaiserslautern, Germany

$\dagger$ Electronic supplementary information (ESI) available: Steady-state absorption and emission spectra of BR at various temperatures and in a MTHF glass at $80 \mathrm{~K}$, multiexponential global fits and target analysis of fs-TA data, semiquantitative analysis of transient absorption anisotropy, time evolution of the fs mid-IR transient spectrum, target analysis of the mid-IR data, a comparison of mid-IR spectra of the $\mathbf{B}_{\text {cold }}$ intermediate with characteristic decay-associated spectra of bacterial phytochromes, time-dependent concentrations of the kinetic model in Scheme 2 and comprehensive description of the experimental materials and methods. See DOI: 10.1039/c5cp06971h
}

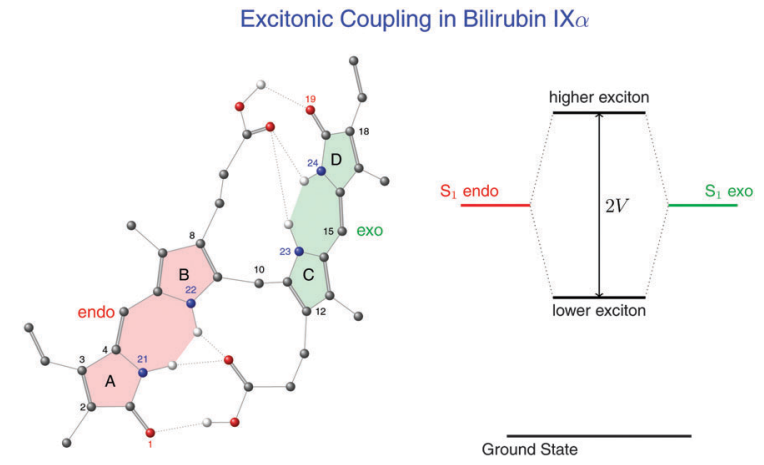

Scheme 1 Three-dimensional structure of bilirubin IX $\alpha$ (left). Dipoledipole coupling $(V)$ of the localized endo and exo $S_{1}$ states explains the lowest bright states of bilirubin (right). $V$ is estimated to be about $500 \mathrm{~cm}^{-1}$.

pyrrinones favours the all-syn $(4 Z, 15 Z)$ "ridge-tile" conformation, ${ }^{10}$ which is however quite flexible at the $\mathrm{C}_{5}-\mathrm{C}_{6}$ and $\mathrm{C}_{14}-\mathrm{C}_{15}$ single bonds and the central methylene linker. This leads to the helical configuration behind the optical activity reported in chiral environments. ${ }^{9,11}$

The lowest excited states of BR are characterized by excitonic coupling of the two dipyrrinone halves, ${ }^{12-15}$ so that the $\pi$-system extends to the whole molecule, despite the loss of conjugation in the central methylene group (Scheme 1). Upon optical excitation, BR deactivates rapidly by internal conversion with almost negligible quantum yields for all other possible deactivation routes at room temperature. ${ }^{11,12,16}$ fs spectroscopy ${ }^{16-18}$ revealed that the deactivation process extends from the 100 fs to the $50 \mathrm{ps}$ time-scales and could be facilitated by a conical intersection in 
the neighbourhood of the Franck-Condon region. ${ }^{19}$ The observation was explained by consecutive mechanisms ${ }^{16,18}$ invoking a "partially twisted" structure from a dark excited state. The latter could be localized in a single dipyrridone moiety, ${ }^{18}$ have charge transfer character, ${ }^{20}$ or result from $\pi \pi^{*}-\mathrm{n} \pi^{*}$ mixing, ${ }^{16}$ but experimental evidence is still lacking.

BR has also attracted considerable interest because of its central role in neonatal jaundice. ${ }^{12}$ Light absorption in the UV region transforms the stable $(4 Z, 15 Z)$ isomer into the polar forms $(4 Z, 15 E)$ and $(4 E, 15 Z)$. The latter show enhanced solubility in blood serum ${ }^{21}$ and can be eliminated in the liver. This is the basic principle of phototherapy ${ }^{22}$ but many details of the isomerisation mechanism remain obscure for various reasons. ${ }^{19,23,24}$ First, the reaction occurs on the early ps timescale with very low quantum yield, about $3 \%$ in solution. ${ }^{12,24-26}$ This limits the spectroscopic study of the forward photoreaction. The backreaction is also hard to analyse because of thermal reversibility of the ground state photoproducts. Second, BR is only weakly soluble in polar and non-polar solvents, which hinders the study of polarity and viscosity effects on the photoreaction. ${ }^{27-31}$ This information was essential to advance in the understanding ${ }^{32,33}$ of the classical $E / Z$ photoisomerisation reactions undergone by stilbenes $^{34-40}$ and azobenzenes. ${ }^{41}$

We report on the deactivation pathway of BR in chloroform by means of broadband fs-resolved optical and mid-IR vibrational spectroscopy. Population dynamics is monitored by fs broadband transient absorption (fs-TA) at room temperature from 270 to $670 \mathrm{~nm}$, i.e. bleach, excited-state absorption (ESA) and stimulated emission (SE) are measured simultaneously with a single laser shot, enabling the independent analysis of spectral diffusion and population evolution. fs-resolved vibrational spectra in the $\mathrm{C}-\mathrm{N}$, $\mathrm{C}=\mathrm{C}$ and $\mathrm{C}=\mathrm{O}$ stretching (str) spectral regions are measured by Vis-pump/mid-IR-probe spectroscopy to identify the reaction intermediate. Optical species-associated-spectra (SAS) are thus connected by a reaction mechanism to characteristic IR resonances, so that the intervening states are best characterized in terms of optical activity and structure. The mechanism is found to be consecutive: the shortlived Franck-Condon state forms a weakly fluorescent intermediate which decays to the ground state with a 15 ps time constant. In contrast to previous proposals, ${ }^{16-18}$ the intermediate shows stronger $\mathrm{C}=\mathrm{C}$ bonds than the ground electronic state, indicating that the molecular structure is not twisted.

\section{Materials and methods}

Bilirubin (BR) was purchased from Acros Organics (99\%) and used as received. Solutions were freshly prepared. Steady-state UV-vis absorption spectra were recorded on a Varian Cary $3 \mathrm{E}$ spectrophotometer. Fluorescence spectra were acquired on a Spex Fluorolog 2 spectrofluorometer at right angles. IR spectra were recorded on a Nicolet 380 FT-IR (Thermo Electron Co.) spectrometer by ATR and transmission.

The fs transient absorption setup at the University of Santiago is analogous to the one described in ref. 42 and 43. Basic pulses were delivered by a multipass Ti:Sa amplifier (Femtolasers
Femtopower Compact Pro, $800 \mathrm{~nm}, 0.8 \mathrm{~mJ}$ per pulse, $30 \mathrm{fs}$, $720 \mathrm{~Hz}$ ). Part of the fundamental beam was frequency doubled in a BBO crystal. The $400 \mathrm{~nm}$ pulses were further divided for optical excitation (typically $0.5 \mu \mathrm{J}$ ) and white-light continuum generation in an oriented $\mathrm{CaF}_{2}$ plate, which was shifted in $X Y$ directions. The white-light was filtered and split for reference before being imaged onto the flowing-sample cell. Transmitted and reference beams were further imaged onto the entrance planes of separate home-made flat-field spectrographs and registered by photodiode arrays. Measurements were performed at parallel and perpendicular pump-probe polarizations. Transient spectra were corrected for the chirp of the white-light continuum. The linear UV-vis absorption spectrum of BR shows no significant changes after fs irradiation.

The fs Vis-pump/mid-IR-probe setup was described elsewhere. ${ }^{44}$ Fundamental pulses were derived from a CPA 2001 (Clark-MXR) regenerative Ti:Sa laser system. It pumps a homebuilt two-stage NOPA tuned in the visible and a two-stage OPA coupled to a $\mathrm{AgGaS}_{2}$ stage for difference frequency generation in the mid-IR. Vis pump pulses were centred at $490 \mathrm{~nm}$. Mid-IR pulses with a typical width of $100 \mathrm{~cm}^{-1}$ were used as the probe. The mid-IR chamber was purged with dry air to avoid pulse lengthening and IR absorption by water. Measurements were performed in three spectral windows centred at 1600, 1645 and $1690 \mathrm{~cm}^{-1}$. The Vis-pump/mid-IR-probe cross-correlation measured in a thin silicon wafer is about 400 fs although the signal fits indicate a shorter cross-correlation. The transient signal is dispersed on a TRIAX 320 polychromator (Jobin Yvon) and imaged onto a 32-element $\mathrm{HgCdTe}$ array at a typical spectral resolution of $2.5 \mathrm{~cm}^{-1}$.

\section{Results and discussion}

\section{1 fs transient absorption in the UV-vis region}

Panel A of Fig. 1 shows the time-evolution of the BR transient absorption spectrum in chloroform from 50 to 500 fs delay. Optical excitation was performed with 30 fs pulses centred at $400 \mathrm{~nm}$. The transient spectrum at $50 \mathrm{fs}$ delay shows the negative contributions of bleach and SE with maxima at about 450 and $560 \mathrm{~nm}$, respectively. Positive contributions from ESA are observed at $500 \mathrm{~nm}$ and to the blue of $400 \mathrm{~nm}$. The spectral evolution right after optical excitation is characterized by (1) the instantaneous red-shift of the SE band, (2) spectral dynamics and (3) decay of SE to form an ESA feature with a double peak structure at about 500 and $600 \mathrm{~nm}$. The instantaneous SE redshift is deduced from the comparison with the first-moment of the SE band in a 2-methyltetrahydrofuran (MTHF) glass at the liquid nitrogen temperature, $\approx 530 \mathrm{~nm}$. This implies an extra reorganization energy of about $500 \mathrm{~cm}^{-1}$ across high-frequency modes at room temperature (Fig. S1 in the ESI $\dagger$ ). Points (2) and (3), fast spectral dynamics and SE decay, are quantified by global analysis in the full spectral window, see Table 1, Fig. 2 and Fig. S2 (ESI $\dagger$ ).

The spectra in Fig. 1A were thus analysed with a biexponential function, a non-decaying component (an offset) and a damped oscillation. A Gaussian function and its first four time-derivatives 


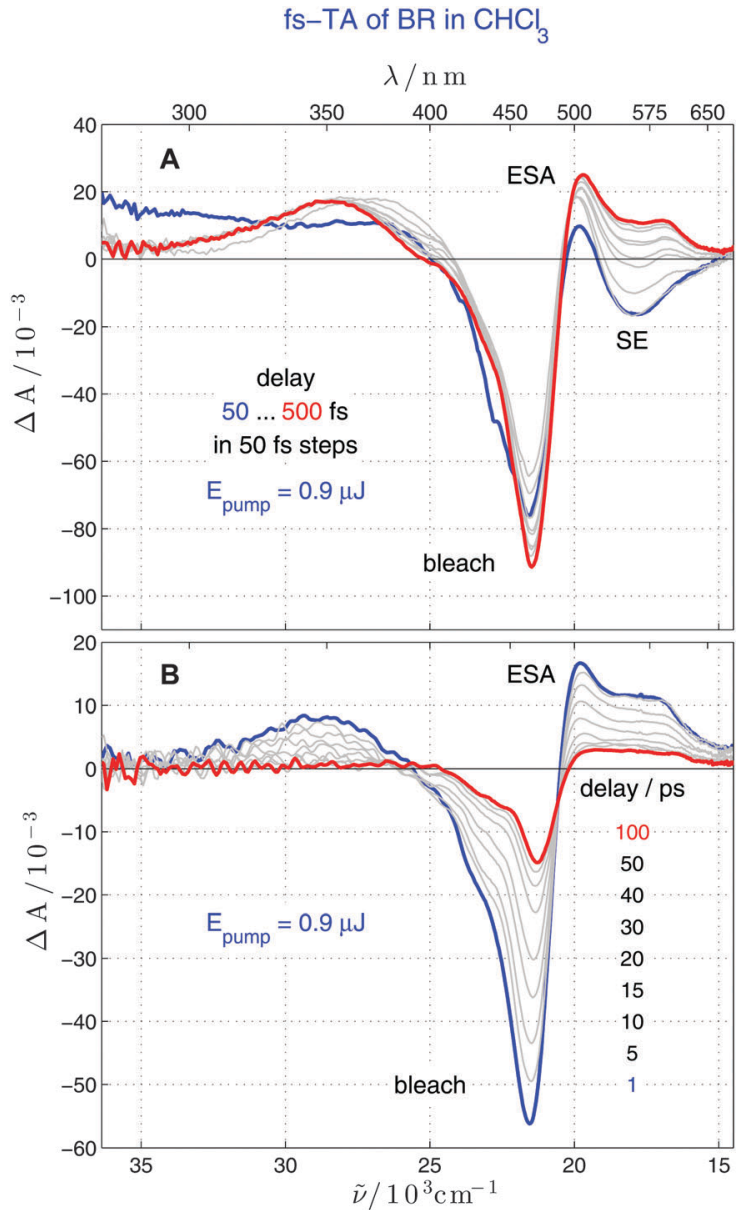

Fig. 1 Spectral evolution of $\mathrm{BR}$ in $\mathrm{CHCl}_{3}$ observed during the earliest $500 \mathrm{fs}$ in the UV-vis spectral window after $400 \mathrm{~nm}$ excitation with parallel pump-probe polarization (panel A). Spectra recorded between 50 (blue) and $500 \mathrm{fs}$ (red) delays are shown in 50 fs steps. SE decays in this time scale. The spectral evolution is not obvious in the bleach and ESA regions because of the underlying coherent contribution and the signal oscillations from a vibrational wavepacket in the solvent (see Fig. S2, ESI †). Panel B shows the spectral evolution in the 100 ps time scale under the same experimental conditions. The time delays are color-coded.

describe the coherent spike around time zero. ${ }^{43}$ The average pump-probe cross-correlation is about $90 \mathrm{fs}$ across the full spectral window. Specifically, it reaches $60 \mathrm{fs}$ in the Vis range and deteriorates in the spectral wings. The fits at representative probe wavelengths are shown in Fig. S2 (ESI $\dagger$ ), whereas the decayassociated-spectra (DAS) are presented in Fig. 2. The exponential functions have optimal decay times of $\tau_{1}=0.06 \mathrm{ps}$ and $\tau_{2}=0.35 \mathrm{ps}$ whereas the oscillatory component shows average values of $368 \mathrm{~cm}^{-1}$ and $0.3 \mathrm{ps}^{-1}$ for the frequency and the damping rate, respectively, both consistent with impulsively stimulated Raman scattering from chloroform. Therefore, the spectral evolution of BR at early time is explained exclusively by the DAS of the 0.06 and 0.35 ps exponentials.

The DAS of $\tau_{1}$ and $\tau_{2}$ are very similar (Fig. 2). They show negative amplitude in the SE region and positive contributions with a double peak structure extending from 350 to $500 \mathrm{~nm}$. A closer look at both spectra shows that the 0.06 ps DAS displays
Table 1 Lifetimes from global analysis of fs broadband transient absorption with multiexponential functions. The number of significant figures takes into account the confidence intervals of the parameters in the fit and the experimental time resolution (20 fs)

\begin{tabular}{llllllll}
$\begin{array}{l}\text { Timescale } \\
(\mathrm{ps})\end{array}$ & $\begin{array}{l}\text { Sampling } \\
\text { rate }(\mathrm{ps})\end{array}$ & $\tau_{1} / \mathrm{ps}$ & $\tau_{2} / \mathrm{ps}$ & $\tau_{3} / \mathrm{ps}$ & $\bar{\tau}_{23}{ }^{a} / \mathrm{ps}$ & $\tau_{4} / \mathrm{ps}$ & $\mathrm{RMS}_{\text {global }} / 10^{-3}$ \\
\hline $1^{b}$ & 0.005 & 0.06 & 0.35 & & & & 0.90 \\
100 & 0.2 & & & & 0.71 & 17.2 & 0.48 \\
$100^{c}$ & 0.2 & & 0.38 & 6.50 & & 14.1 & 0.44 \\
300 & 0.5 & & & & 0.61 & 16.5 & 0.44 \\
1500 & 2 & & & & 0.6 & 16.7 & 0.84 \\
$100\left(\mathrm{IR}^{d}\right)$ & & & & & 1.5 & 12 &
\end{tabular}

${ }^{a} \tau_{2}$ and $\tau_{3}$ collapse into $\bar{\tau}_{23}$ at slow sampling rates. ${ }^{b}$ Fitting function contains a damped oscillation of the type $\mathrm{e}^{-\gamma t} \cos \left\{\left(\omega t+\varphi\left(\lambda_{\text {probe }}\right)\right)\right\}$, with average values of $\bar{\omega}=368 \mathrm{~cm}^{-1}$ and $\bar{\gamma}=0.3 \mathrm{ps}^{-1}$. ${ }^{c}$ Tri-exponential fit. ${ }^{d}$ In the fs mid-IR experiment the sampling rate was varied across the delay line.

narrower bands at 394 and $550 \mathrm{~nm}$. In turn, the ESA feature at $465 \mathrm{~nm}$ has a FWHM of $\approx 2000 \mathrm{~cm}^{-1}$ in the $0.06 \mathrm{ps}$ DAS but shifts to the red and narrows in the 0.35 ps DAS, where the peak is located at $470 \mathrm{~nm}$ and the FWHM decreases to $1400 \mathrm{~cm}^{-1}$. Next, the negative amplitudes in the SE band obviously explain the observed decay of SE in the sub-ps time scale with no spectral shift. Therefore, the positive amplitude of the DAS overlapping the bleach region can only arise from the decay of ESA-like bands peaking at about 400 and $460 \mathrm{~nm}$. Otherwise one would have to assume that the bleach rises slower than instrumental crosscorrelation, which is unphysical. Consequently, the fastest time

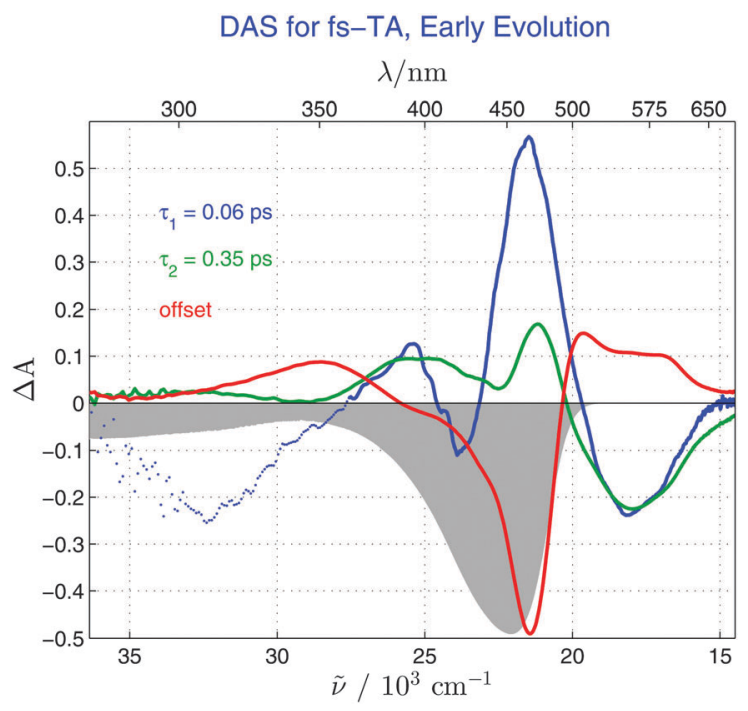

Fig. 2 Decay associated spectra (DAS) obtained from the fits in Fig. S2 $(E S \mid+)$. Decay times of 0.06 and 0.35 ps were deduced. The amplitude of the faster component is uncertain to the blue of $350 \mathrm{~nm}$ (blue dotted line) because of the longer cross-correlation in the UV region. Note that the global fit procedure assumes a constant cross-correlation across the spectral window. This assumption distorts spectra associated with ultrafast decay times in the UV and near-IR regions, where the instrumental crosscorrelation is broader. Hence, the DAS of the 0.06 ps component are unreliable to the blue of $350 \mathrm{~nm}$ and to the red of $650 \mathrm{~nm}$. The steadystate absorption spectrum of BR is shown as an inverted grey-filled curve for comparison. 
constants account for excited-state processes only because their contribution to the bleach signal is negligible. We also interpret the close resemblance of the 0.06 and 0.35 ps DAS as a signature of spectral dynamics in the the Franck-Condon state rather than the conversion between excited states. Thus, the spectrum of the Franck-Condon state develops with a characteristic time constant of 0.06 ps as a consequence of BR wavepacket spreading and dephasing in the course of intramolecular vibrational redistribution (IVR) and relaxation. Short population and dephasing times overdamp signal recurrences from BR. The subsequent decay of SE monitors the excursion from the relaxed Franck-Condon state A to a new excited state B. The latter is weakly fluorescent because the fs-TA at 1 ps delay shows no clear indication of SE, except for the ESA dip around $560 \mathrm{~nm}$ that is understood as a signature of SE with very weak cross section.

Fluorescence up-conversion measurements demonstrate that the fluorescence signal of BR decays in multiple time scales in chloroform and bound to human serum albumin in water. ${ }^{17,18}$ Time constants of 0.134 (51\%), 0.58 (37\%), 2.2 (9\%) and 9.4 ps $(3 \%)$ were reported in these publications, resulting in a characteristic fluorescence decay time of about $0.8 \mathrm{ps}$ in chloroform. The above-discussed fs-TA measurements however show that early dynamics, characterized by time constants faster than $0.5 \mathrm{ps}$, does not imply the decay of bleach. This means first that the process $\mathbf{A} \rightarrow \mathbf{B}$ discussed above involves excited-state population only and accounts for more than $35 \%$ of the emitted photons, as estimated from the integral over time of the fluorescence decay envelope. The ultrafast components of the fluorescence decay do not arise from a decay of population to the ground state but from a wavepacket propagating from the bright state A to state B. Second and surprisingly, about $65 \%$ of the fluorescence emission stems from the weakly fluorescent state $\mathbf{B}$, which simply emits longer. We remark that our global analysis empirically models the spreading of the wavepacket in the Franck-Condon potential well and its approach to the crossing region with state $\mathbf{B}$. The latter could borrow the oscillator strength for SE from the bright excitonic state $\mathbf{A}$.

The fs-TA spectrum also evolves in the 100 ps time scale, Fig. 1B, where bleach recovery indicates that the excited-state population replenishes the ground state. Simultaneously, the ESA band at around $550 \mathrm{~nm}$ turns its double peak structure at 1 ps delay into a broad ESA band extending from 500 to $650 \mathrm{~nm}$, which probably reflects how the SE cross-section decreases in state $\mathbf{B}$ as it relaxes further.

We address now the global analysis of the transient absorption spectra at long delays, see Table 1 and Fig. S3, S4 (ESI $\dagger$ ). Time constants of $(0.65 \pm 0.05)$ and $(15 \pm 2)$ ps are deduced from the biexponential fits in Table 1. The global RMS values indicate however a better performance of the tri-exponential fit, from which time constants of $0.38,6.50$ and 14.1 ps result in the 100 ps window. The sampling rate of this experiment is just enough for the simultaneous resolution of the three time constants, although it is too coarse to sense the $0.06 \mathrm{ps}$ time constant associated with the relaxation of the Franck-Condon state. The $0.38 \mathrm{ps}$ time constant is consistent with the previously discussed conversion between states $\mathbf{A}$ and $\mathbf{B}$, whereas the 6.50 ps lifetime accounts for small spectral changes as expected from the spectral evolution in Fig. 1B and the DAS in Fig. S3 (ESI $\dagger$ ). Thus, we assign the $6.50 \mathrm{ps}$ time to vibrational cooling in the $\mathbf{B}$ excited state. It may be accompanied by a decrease of the $\mathbf{A}-\mathbf{B}$ coupling strength and the SE cross-section of state $\mathbf{B}$. We name the relaxed $\mathbf{B}$ state as $\mathbf{B}_{\text {cold. }}$. The latter decays to the ground state with $a \approx 15$ ps time constant, which is associated with the decay of bleach and the ESA bands at 350 and $\approx 550 \mathrm{~nm}$, respectively.

\subsection{Two-photon excitation}

We clearly observe that high pump energies induce an increase of the long-living contribution on the 250 ps time-scale (Fig. 3). Pump energy was varied between 0.3 and $1.7 \mu \mathrm{J}$ with a neutral density filter so that minimal changes on pump-probe overlap occur. The photodynamics is quantified by means of band integrals (BI) in the bleach and ESA regions for a better signal-to-noise ratio. A biexponential function with a non-decaying component describes very well the experimental BIs in this time scale, where the short exponential ( $c a .15 \mathrm{ps)} \mathrm{accounts} \mathrm{for} \mathrm{the} \mathrm{decay} \mathrm{to} \mathrm{the} \mathrm{ground} \mathrm{state}$ whereas the long exponential ( $c a .200 \mathrm{ps)}$ arises from rotational diffusion of the non-decaying species in the excited state, Fig. 3A. The value of the rotational diffusion time constant is quite reasonable for a molecule of this size and is corroborated by semiquantitative analysis of signal anisotropy (see ESI $\dagger$ ). The amplitudes at time zero are analysed in Fig. 3B, where we define $A_{\mathrm{E}}$ as the time-zero amplitude of the short exponential and $A_{\mathrm{O}}$ as the sum of the time-zero amplitudes of the long exponential and the offset. The ratios $\frac{A_{\mathrm{O}}}{A_{\mathrm{E}}+A_{\mathrm{O}}}$ calculated for the bleach and ESA bands depend linearly on pump energy. This in turn implies a quadratic dependence of $A_{\mathrm{O}}$ because $A_{\mathrm{E}}+A_{\mathrm{O}}$ is quasi-linear. Therefore, our analysis suggests that the non-decaying component is generated by coherent two-photon excitation. A sequential twophoton mechanism seems less plausible in view of the low ESA cross-section at $400 \mathrm{~nm}$. We note in passing that the use of the amplitude ratio $\frac{A_{\mathrm{O}}}{A_{\mathrm{E}}+A_{\mathrm{O}}}$ to characterize the two-photon process shows important advantages versus the amplitude of the longliving component alone, for which a quadratic dependence is expected. First and most important, the amplitude ratio does not depend on sample concentration. The band integrals in Fig. 3 are an average of several scans, typically five, performed on independent samples for each pump energy. While this is essential to measure the offset contribution accurately especially at low pump energies and to ensure sample photostability, it introduces noise in the absolute amplitude of the one- and twophoton components because of the varying BR concentrations. Second, it can be shown that the slope of the bleach and ESA plots in Fig. 3B must be the same, as observed, and reports the ratio between the two- and one-photon excitation cross-sections at $400 \mathrm{~nm}, \approx 10 \%$. Finally, we estimate an offset contribution of about $25 \%$ for an instantaneous intensity of $0.5 \mathrm{TW} \mathrm{cm}^{-2}$. This is easily achievable with fs pulses but translates into very harsh conditions for $\mathrm{CW}$ sources. Consequently, the generation of BR photoproducts under ambient-light conditions or with CW sources must occur by one-photon excitation with very low yield, 

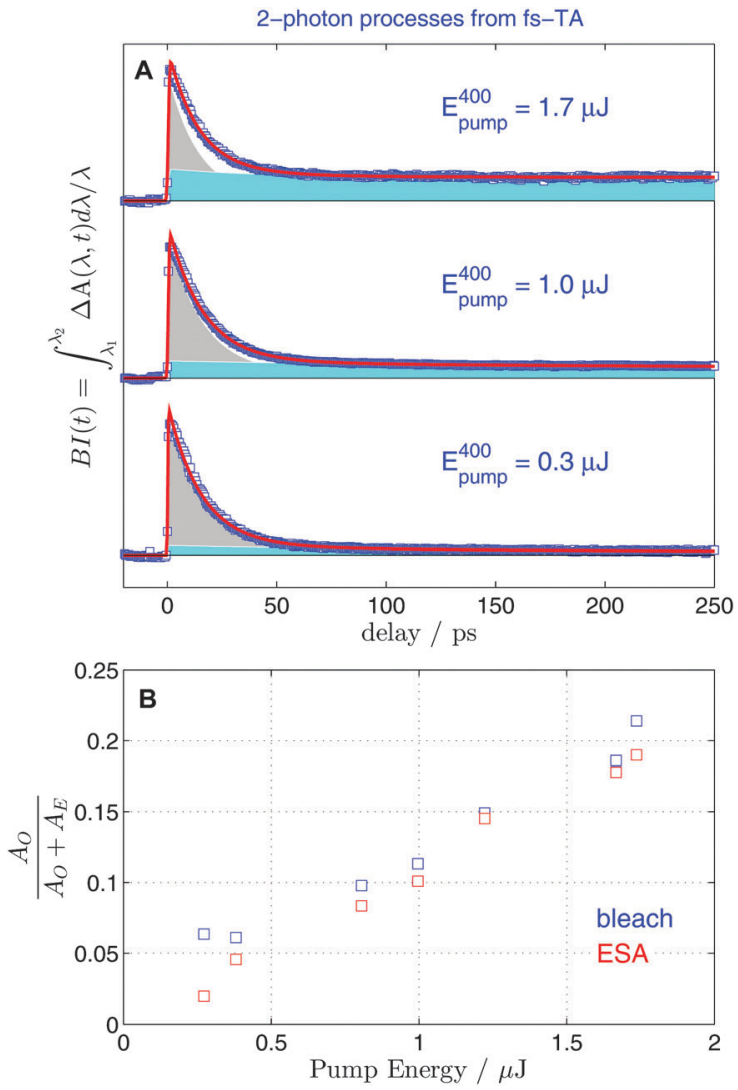

Fig. 3 Panel A shows the normalized bleach band integrals calculated between 415 and $475 \mathrm{~nm}$ at the indicated pump energies (blue squares). The pump-probe polarization is parallel. The fit with a biexponential function and a non-decaying component is shown in red. The fast exponential, ca. $15 \mathrm{ps}$, is shown as a gray-filled curve whereas the contributions of the long exponential ( $\approx 200 \mathrm{ps,} \mathrm{due} \mathrm{to} \mathrm{rotational} \mathrm{diffusion)}$ and the offset are quantified by the cyan-filled curve. Panel B shows how the relative amplitudes of the long-living components depend on pump energy. They are calculated as the ratio between the time-zero amplitudes of the long exponential (accounting for rotational diffusion) and the offset $\left(A_{\circ}\right)$ and the full amplitude $A_{O}+A_{E}$, where $A_{E}$ is the time-zero amplitude of the fast exponential. The blue squares show the calculations for the bleach band while the red squares refer to the ESA band at about $600 \mathrm{~nm}$. The ESA band integral is calculated from 525 to $625 \mathrm{~nm}$.

less than $3 \%{ }^{23,24,45}$ The yield is so low that no photoproducts could be detected for pump fluencies at the focal waist below $4 \mathrm{~mJ} \mathrm{~cm}^{-2}$ (0.3 $\mu \mathrm{J}$ pump energy), for which the two-photon contribution is negligible. In turn, the two-photon contribution rapidly leaves the one-photon part behind as the pump energy is increased.

We summarize the interpretation of the UV-vis transient absorption measurements and propose the deactivation mechanism. Optical excitation populates the Franck-Condon state, which rapidly relaxes by IVR to state $\mathbf{A}$. The latter couples to state $\mathbf{B}$, which is weakly fluorescent, and relaxes to $\mathbf{B}_{\text {cold }}$ by vibrational cooling. The initial structure of the ground state is repopulated from there with a characteristic time constant of about 15 ps and an efficiency close to $100 \%$. In turn, two-photon excitation at $400 \mathrm{~nm}$ leads to the nondecaying component, C. Note however that the transient spectrum of the non-decaying component (cyan spectrum in Fig. S3, ESI $\dagger$ )

\section{One-photon mechanism}

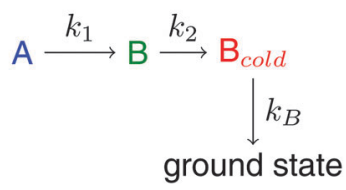

Scheme 2 Mechanism for the deactivation of BR after 1-photon excitation.

differs somehow from the absorbance difference spectrum of the photoequilibrated BR sample published in ref. 23 and Fig. S5 (ESI†). This indicates that $\mathbf{C}$ could be a precursor of the $(4 Z, 15 E)$ major isomer. The mechanism is shown in Scheme 2 for the one-photon processes only. The resulting time-dependent concentrations are deduced in the ESI. $\dagger$ The species-associated spectra (SAS) of the intervening species are obtained by global target analysis with the time-dependent concentrations derived from the mechanism (Fig. 4). The SAS represent the UV-vis linear difference absorption spectra of the transient species involved. Optimal values of the microscopic rate constants are collected in Table 2, whereas the global target fits are shown in Fig. S6 (ESI $\dagger$ ).

\section{3 fs transient absorption in the mid-IR region}

Fig. 5 focuses on early stages of BR deactivation as monitored by ultrafast spectroscopy in the mid-IR range with optical excitation at $490 \mathrm{~nm}$. The experiment probes the spectral evolution of $\mathrm{C}-\mathrm{N}, \mathrm{C}=\mathrm{C}$ and $\mathrm{C}=\mathrm{O}$ str vibrations, which were addressed independently in spectral windows centred at 1600 , 1645 and $1690 \mathrm{~cm}^{-1}$, respectively. The windows were joined and smoothed with a Savitzky-Golay filter for better visibility in

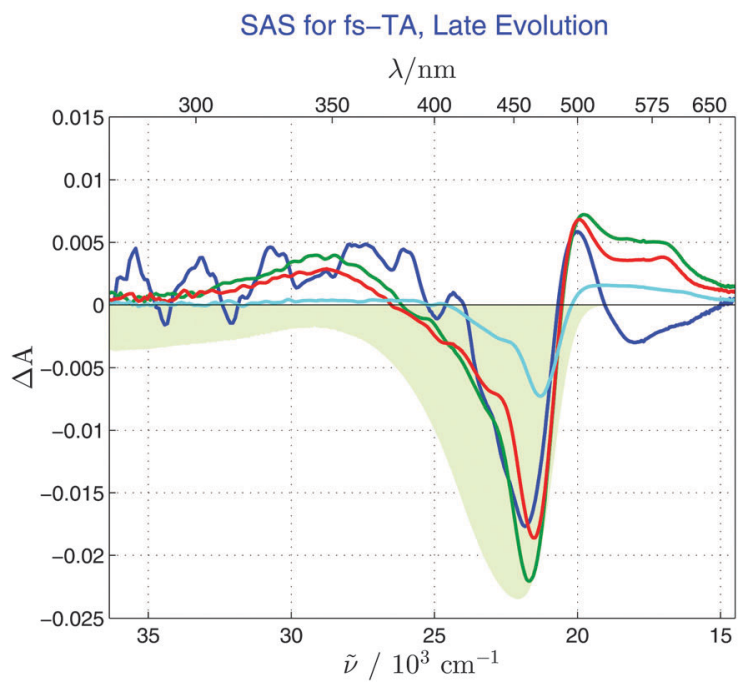

Fig. 4 SAS obtained from the kinetic analysis in the 100 ps time scale with constant steps of $200 \mathrm{fs}$. Ultrafast wavepacket spreading and IVR in state $\mathbf{A}$ go unnoticed in this time scale (A, blue). B (green) relaxes to $\mathbf{B}_{\text {cold }}$ (red) mainly by vibrational cooling. $\mathbf{C}$ (cyan) is the long-living component that forms by 2-photon excitation in the fs experiments. The mechanism for one-photon excitation, from which $\mathbf{A}, \mathbf{B}$ and $\mathbf{B}_{\text {cold }}$-SAS derive, is shown in Scheme 2. Optimal values of the associated rate constants are collected in Table 2. 
Table 2 Rate constants obtained from the target global analysis of fs-TA and fs-IR data

\begin{tabular}{llllll}
\hline Dataset & $k_{1} / \mathrm{ps}^{-1}$ & $k_{2} / \mathrm{ps}^{-1}$ & $\bar{k}_{12} / \mathrm{ps}^{-1}$ & $k_{\mathrm{B}} / \mathrm{ps}^{-1}$ & $\mathrm{IRF} / \mathrm{ps}$ \\
\hline fs-TA & 2.53 & 0.14 & & 0.07 & $0.1^{a}$ \\
fs-IR & & & $0.65^{b}$ & 0.08 & 0.2
\end{tabular}

${ }^{a}$ FWHM of the pump-probe cross-correlation for the global target analysis with a step size of $200 \mathrm{fs} .{ }^{b}$ The fast components are resolved only partially in the IR experiment, where $\bar{k}_{12}$ averages out $k_{1}$ and $k_{2}$ from fs-TA.

Fig. 5 but raw data were employed for further analysis. Only minor changes are observed. The earliest two ps are characterised by the increasingly negative amplitude of the $\mathrm{C}=\mathrm{C}$ and $\mathrm{C}-\mathrm{N}$ bands and by the blue-shift and spectral narrowing of the $\mathrm{C}=\mathrm{O}$ ESA feature at around $1700 \mathrm{~cm}^{-1}$. The signal decays with constant spectral shape at longer delays (Fig. S9, ESI $\dagger$ ). In the following, global analysis in time domain helps to identify the underlying structural changes.

A biexponential global fit yields time constants of 1.5 and 12 ps (Table 1), in good agreement with the UV-vis analysis when the lower time-resolution and signal-to-noise of the mid-IR experiment is considered. The mechanism therefore reduces to $\mathbf{A B} \stackrel{\bar{k}_{12}}{\longrightarrow} \mathbf{B}_{\text {cold }} \stackrel{k_{\mathrm{B}}}{\longrightarrow}$, where $\bar{k}_{12}$ is an average rate constant accounting for the processes with rate constants $k_{1}$ and $k_{2}$ and $\mathbf{A B}$ is an average over time of the $\mathbf{A}$ and $\mathbf{B}$ individual structures. The latter could not be resolved by us in the mid-IR experiment. A target analysis with the resulting time-dependent concentrations leads to the SAS spectra in Fig. 6 (see also Fig. S10 in the ESI $\dagger$ ).

The spectral features in Fig. 6 are assigned with the aid of earlier IR measurements on $\mathrm{BR}^{46,47}$ and other studies ${ }^{48,49}$ addressing the photoreactions of bilin chromophores in bacterial phytochromes. Most remarkably, the transition from the ground

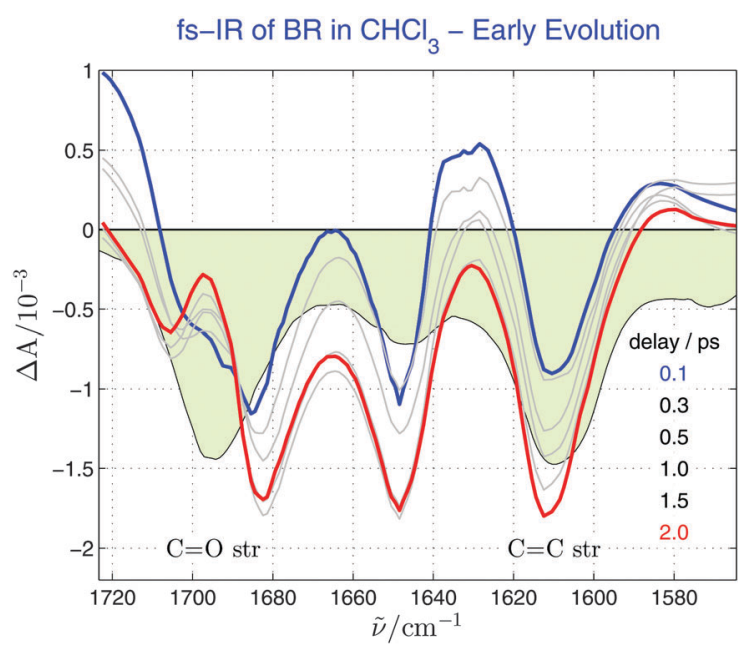

Fig. 5 Vis-pump/mid-IR probe transient absorption spectra of BR in chloroform at the indicated pump-probe delays in the $\mathrm{C}-\mathrm{N}, \mathrm{C}=\mathrm{C}$ and $\mathrm{C}=\mathrm{O}$ str regions. The steady-state mid-IR absorption spectrum is shown as a light-green-filled curve. Transient spectra were measured in three independent windows (1580-1635, 1610-1680 and 1650-1730 cm ${ }^{-1}$ ), joined and smoothed with a Savitzki-Golay filter for better visibility. Early spectral evolution is shown here. state to the $\mathbf{A B}$ state is characterized by frequency up-shifts of the $\mathrm{C}-\mathrm{N}, \mathrm{C}=\mathrm{C}, \mathrm{C}_{15}=\mathrm{C}_{16}$ and $\mathrm{C}_{19}=\mathrm{O}$ str modes with ground-state resonances at 1570, 1610, 1645 and $1695 \mathrm{~cm}^{-1}$, respectively. The $\mathrm{C}=\mathrm{C}$ str modes show frequency up-shifts of about $20 \mathrm{~cm}^{-1}$. Their absorption cross-section seems to be lower in the $\mathbf{A B}$ state compared to the ground state as suggested by the negative amplitude of the transient signal in the $\mathrm{C}=\mathrm{C}$ spectral window. In addition, the ground-state to $\mathbf{A B}$ up-shifts are more modest in the case of the $\mathrm{C}-\mathrm{N}$ and $\mathrm{C}_{19}=\mathrm{O}$ str vibrations. They amount to 15 and $2 \mathrm{~cm}^{-1}$, respectively. Relaxation to $\mathbf{B}_{\text {cold }}$ brings about an additional up-shift of $3-5 \mathrm{~cm}^{-1}$ in the $\mathrm{C}=\mathrm{C}, \mathrm{C}_{15}=\mathrm{C}_{16}$ and the $\mathrm{C}_{19}=\mathrm{O}$ modes, which is compatible with the suggested vibrational cooling in the excited state surface. Simultaneously, the C-N str mode down-shifts by $3 \mathrm{~cm}^{-1}$, indicating that the intramolecular hydrogen bond weakens at the $\mathrm{C}_{19}=\mathrm{O}$ and strengthens at the $\mathrm{N}-\mathrm{H}$ site in the course of vibrational cooling.

Altogether, our fs experiments demonstrate that the major deactivation route of BR occurs through the stable species $\mathbf{B}_{\text {cold }}$. The latter shows tighter skeletal bonds than the ground state. This observation is difficult to reconcile with the assumption of a molecular structure partially "twisted" around the $\mathrm{C}_{15}=\mathrm{C}$ bond, ${ }^{16-19}$ for which a frequency down-shift of the $\mathrm{C}=\mathrm{C}$ str

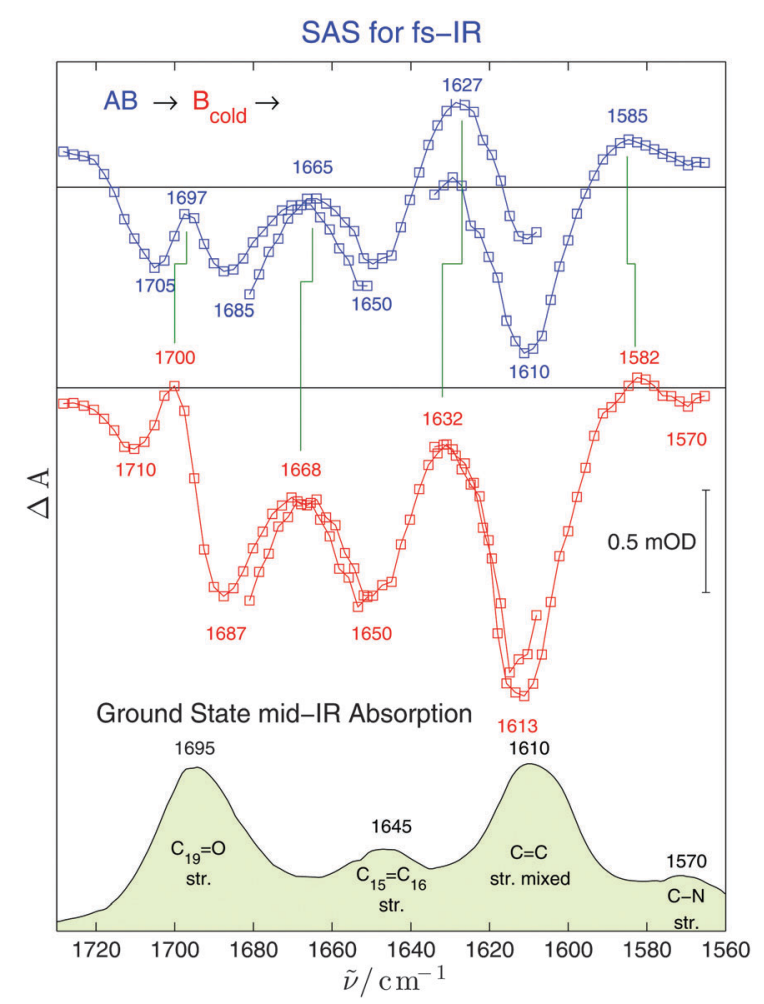

Fig. 6 SAS obtained for the Vis-pump/mid-IR probe transient absorption spectra of BR in chloroform after the mechanism in the inset of Fig. 3, as seen with the time resolution of the mid-IR experiment. The $\mathrm{C}-\mathrm{N}, \mathrm{C}=\mathrm{C}$ and $\mathrm{C}=\mathrm{O}$ str regions were studied. The steady-state mid-IR absorption spectrum is shown as a light-green-filled curve. Transient spectra were recorded in three independent windows (1580-1635, 1610-1680 and 1650-1730 $\mathrm{cm}^{-1}$ ) and fitted globally by target analysis. The green lines show the shift of the excited-state signals during the $\mathbf{A B}$ (blue) to $\mathbf{B}_{\text {cold }}$ (red) transformation. A tentative band assignment of the steady-state mid-IR spectrum is indicated. 
modes is expected. Yet, one cannot exclude that "twisted" molecular structures mediate the one-photon $Z, E$ photoisomerization of BR, which shows however a much minor yield and still escapes detection.

To close, we compare BR behaviour with the ultrafast dynamics of the bilin chromophores in phytochromes. BR fs-to-ps dynamics is very close to that observed for phytochromes, ${ }^{50,51}$ small protein photoreceptors binding bilin chromophores, which isomerize at the $\mathrm{C}_{15}$ position too. A double bond at $\mathrm{C}_{9}-\mathrm{C}_{10}$ and the specific interactions with the surrounding amino acids and protein-bound water molecules constitute the main structural differences between the protein-bound bilins and BR. This leads to a larger "pitch" in the helical structure of BR and a concomitant blue-shift of the UV-vis absorption spectrum. Despite this, the $\mathbf{B}_{\text {cold }}$ mid-IR spectrum closely resembles those obtained for the 15-30 ps decay components observed in the photocycles of bacterial phytochromes from Agrobacterium tumefaciens ${ }^{48}$ and Cyanobacterium syneccocystis $^{49}$ (Fig. S11, ESI $\dagger$ ). The observation is more staggering when one underlines that the phytochrome spectra shown in Fig. S11 (ESI $\dagger$ ) are decay-associated whereas the BR ones are species-associated. Eqn (S2) (ESI $\dagger$ ) demonstrates that the coincidence between both kinds of spectra is only expectable if both molecules follow a similar mechanism. The mechanism proposed in ref. 48 is indeed very similar to the one deduced here. Therefore, the marked parallelism between characteristic time scales, the molecular structure of the dark intermediate and the $Z, E$ isomerization site of the protein-bound bilins and BR suggest that both chromophores could stick to the same underlying mechanism.

\section{Conclusions}

Ultrafast deactivation of BR occurs via the dark intermediate $\mathbf{B}_{\text {cold }}$. The latter decays to the ground state with a 15 ps time constant. The mid-IR spectrum of $\mathbf{B}_{\text {cold }}$ points to a stiff molecular skeleton that does not easily agree with the "twisted" structure conjectured by several authors so far. ${ }^{16-19}$ Thus, BR deactivation occurs via high-frequency coordinates with a yield close to $100 \%$. We found indication for two-photon enhancement of the otherwise minor $Z, E$ isomerization channel, which might occur by the $\mathrm{C}-\mathrm{C}$ torsion via a long-living intermediate. It remains open which electronic states are behind the intermediates of the one- and two-photon processes. Low-lying dark charge-transfer states computed by quantum chemical methods ${ }^{20}$ would be the most obvious candidates for $\mathbf{B}_{\text {cold }}$, but they are expected to induce strong dynamic solvation shifts in the SE band, ${ }^{52}$ contrary to observation. Spectroscopy-guided high-level quantum chemical calculations are the most natural way to the answer. Finally, the agreement observed among the photoreactions of various open tetrapyrroles emphasizes the role of elusive intermediates as ultrafast regulators of signalling processes in biology.

\section{Acknowledgements}

JLPL thanks the Spanish Ministry of Science and Innovation (MICINN) for funding through the grant CTQ2010-17026
(FEDER Funds) and the "Ramón y Cajal" Program 2009, as well as the Xunta de Galicia (Spain) for grants EM2012/091, GPC2013/052 and R2014/051. CCB thanks the Spanish Ministry of Education for a FPU doctoral grant. We thank Prof. M. Flor Rodríguez Prieto and Manuel Mosquera (University of Santiago, Spain) for support and Hans Feurich and Fabian Rupp (University of Kaiserslautern) for technical assistance. RD and PS acknowledge financial support from Research Initiative Rheinland Pfalz (BioComp).

\section{References}

1 N. C. Rockwell and J. C. Lagarias, ChemPhysChem, 2010, 11, 1172-1180.

2 R. D. Vierstra and J. Zhang, Trends Plant Sci., 2011, 16, 417-426.

3 M. R. Wasielewski, Chem. Rev., 1992, 92, 435-461.

4 D. Gust, T. A. Moore and A. L. Moore, Acc. Chem. Res., 2009, 42, 1890-1898.

5 L.-L. Li and E. W.-G. Diau, Chem. Soc. Rev., 2013, 42, 291-304.

6 E. S. Nyman and P. H. Hynninen, J. Photochem. Photobiol., B, 2004, 73, 1-28.

7 T. W. Sedlak and S. H. Snyder, Pediatrics, 2004, 113, 1776-1782.

8 G. Le Bas, A. Allegret, Y. Mauguen, C. Derango and M. Bailly, Acta Crystallogr., Sect. B: Struct. Crystallogr. Cryst. Chem., 1980, 36, 3007-3011.

9 R. V. Person, B. R. Peterson and D. A. Lightner, J. Am. Chem. Soc., 1994, 116, 42-59.

10 R. Bonnett, J. E. Davies and M. B. Hursthouse, Nature, 1976, 262, 327-328.

11 S. E. Braslavsky, A. R. Holzwarth and K. Schaffner, Angew. Chem., Int. Ed., 1983, 22, 656-674.

12 G. Agati and F. Fusi, J. Photochem. Photobiol., B, 1990, 7, 1-14.

13 G. J. Troup, G. Agati, F. Fusi and R. Pratesi, Aust. J. Phys., 1996, 49, 673-681.

14 M. Mazzoni, G. Agati, G. J. Troup and R. Pratesi, J. Opt. A: Pure Appl. Opt., 2003, 5, 374-380.

15 M. Mazzoni, G. Agati, R. Pratesi and M. Persico, J. Opt. A: Pure Appl. Opt., 2005, 7, 742-747.

16 B. I. Greene, A. A. Lamola and C. V. Shank, Proc. Natl. Acad. Sci. U. S. A., 1981, 78, 2008-2012.

17 B. Zietz, A. N. Macpherson and T. Gillbro, Phys. Chem. Chem. Phys., 2004, 6, 4535-4537.

18 B. Zietz and T. Gillbro, J. Phys. Chem. B, 2007, 111, 11997-12003.

19 B. Zietz and F. Blomgren, Chem. Phys. Lett., 2006, 420, 556-561.

20 G. Granucci, M. Mazzoni, M. Persicoa and A. Tonioloa, Phys. Chem. Chem. Phys., 2005, 7, 2594-2598.

21 A. F. McDonagh and D. A. Lightner, Pediatrics, 1985, 75, 443-455. 22 J. F. Ennever, Photochem. Photobiol., 1988, 47, 871-876.

23 D. A. Lightner, T. A. Wooldridge and A. F. McDonagh, Proc. Natl. Acad. Sci. U. S. A., 1979, 76, 29-32.

24 A. F. McDonagh, G. Agati and D. A. Lightner, Monatsh. Chem., 1998, 129, 649-660.

25 A. F. McDonagh, L. A. Palma and D. A. Lightner, Science, 1980, 208, 145-151. 
26 A. F. McDonagh, L. A. Palma and D. A. Lightner, J. Am. Chem. Soc., 1982, 104, 6867-6869.

27 S. Abrash, S. Repinec and R. M. Hochstrasser, J. Chem. Phys., 1990, 93, 1041-1053.

28 D. C. Todd, J. M. Jean, S. J. Rosenthal, A. J. Ruggiero, D. Yang and G. R. Fleming, J. Chem. Phys., 1990, 93, 8658-8668.

29 L. Nikowa, D. Schwarzer, J. Troe and J. Schroeder, J. Chem. Phys., 1992, 97, 4827-4835.

30 D. C. Todd and G. R. Fleming, J. Chem. Phys., 1993, 98, 269-279.

31 R. J. Sension, S. T. Repinec, A. Z. Szarka and R. M. Hochstrasser, J. Chem. Phys., 1993, 98, 6291-6315.

32 R. S. H. Liu and G. S. Hammond, Proc. Natl. Acad. Sci. U. S. A., 2000, 97, 11153-11158.

33 D. Sampedro Ruiz, A. Cembran, M. Garavelli, M. Olivucci and W. Fuss, Photochem. Photobiol., 2002, 76, 622-633.

34 D. H. Waldeck, Chem. Rev., 1991, 91, 415-436.

35 J. S. Baskin, L. Banares, S. Pedersen and A. H. Zewail, J. Phys. Chem., 1996, 100, 11920-11933.

36 W. Fuss, C. Kosmidis, W. Schmid and S. Trushin, Chem. Phys. Lett., 2004, 385, 423-430.

37 S. A. Kovalenko, A. L. Dobryakov, I. Ioffe and N. P. Ernsting, Chem. Phys. Lett., 2010, 493, 255-258.

38 S. A. Kovalenko, A. L. Dobryakov, E. Pollak and N. P. Ernsting, J. Chem. Phys., 2013, 139, 011101.

39 S. A. Kovalenko and A. L. Dobryakov, Chem. Phys. Lett., 2013, 570, 56-60.
40 M. Quick, F. Berndt, A. L. Dobryakov, I. N. Ioffe, A. A. Granovsky, C. Knie, R. Mahrwald, D. Lenoir, N. P. Ernsting and S. A. Kovalenko, J. Phys. Chem. B, 2014, 118, 1389-1402.

41 M. Quick, A. L. Dobryakov, M. Gerecke, C. Richter, F. Berndt, I. N. Ioffe, A. A. Granovsky, R. Mahrwald, N. P. Ernsting and S. A. Kovalenko, J. Phys. Chem. B, 2014, 118, 8756-8771.

42 A. L. Dobryakov, S. A. Kovalenko, A. Weigel, J. L. PerezLustres, J. Lange, A. Mueller and N. P. Ernsting, Rev. Sci. Instrum., 2010, 81, 113106.

43 S. A. Kovalenko, A. L. Dobryakov, J. Ruthmann and N. P. Ernsting, Phys. Rev. A: At., Mol., Opt. Phys., 1999, 59, 2369-2384.

44 F. Peters, J. Herbst, J. Tittor, D. Oesterhelt and R. Diller, Chem. Phys., 2006, 323, 109-116.

45 M. G. Migliorini, P. Galvan, G. Sbrana, G. P. Donzelli and C. Vecchi, Biochem. J., 1988, 256, 841-846.

46 A. K. Rai, S. B. Rai, D. K. Rai and V. B. Singh, Spectrochim. Acta, Part A, 2002, 58, 2145-2152.

47 N. Suzuki and M. Toyoda, Tohoku J. Exp. Med., 1966, 88, 353-360.

48 C. Schumann, R. Gross, N. Michael, T. Lamparter and R. Dilier, ChemPhysChem, 2007, 8, 1657-1663.

49 J. J. van Thor, L. Ronayne and M. Towrie, J. Am. Chem. Soc., 2007, 129, 126-132.

50 M. Bischoff, G. Hermann, S. Rentsch and D. Strehlow, Biochemistry, 2001, 40, 181-186.

51 K. Heyne, J. Herbst, D. Stehlik, B. Esteban, T. Lamparter, J. Hughes and R. Diller, Biophys. J., 2002, 82, 1004-1016.

52 M. L. Horng, J. A. J. A. Gardecki, A. Papazyan and M. Maroncelli, J. Phys. Chem., 1995, 99, 17311-17337. 